\title{
Desarrollo de un modelo empírico para la predicción estacional sobre el Mediterráneo
}

https://doi.org/10.31978/639-19-010-0.629

\author{
Esteban Rodríguez Guisado' (erodriguezg@aemet.es) \\ Antonio Ángel Serrano de la Torre ${ }^{1}$ (aserranot@aemet.es) \\ Eroteida Sánchez García² (esanchezg@aemet.es) \\ Beatriz Navascués Fernández-Victorio ${ }^{3}$ (bnavascuesf@aemet.es) \\ Ernesto Rodríguez Camino ${ }^{1}$ (erodriguezc@aemet.es) \\ ${ }^{1} A E M E T /$ Dirección de Producción e Infraestructuras \\ ${ }^{2}$ AEMET / Delegación Territorial en Cantabria \\ ${ }^{3}$ AEMET / Dirección de Estrategia, Planificación y Desarrollo Comercial
}

\begin{abstract}
RESUMEN
Se propone el desarrollo de un modelo empírico de predicción que complete la información proporcionada por los sistemas basados en modelos dinámicos. Está basado en regresión lineal múltiple, usando la temperatura promedio en superficie y la precipitación acumulada como predictandos e índices climáticos globales como predictores. Su implementación permite modificar la lista de predictores de forma sencilla, lo que permitirá incorporar la información de nuevos índices que se obtengan de los experimentos de sensibilidad actualmente en curso realizados con modelos en el marco del proyecto MEDSCOPE.

Dada la extensión del área estudiada y su gran complejidad cada zona se ve afectada por diferentes factores en diferentes momentos del año. Por ello, se ha dividido el área geográfica en estudio en diez regiones, en cada una de las cuales el modelo utiliza diferentes grupos de predictores, que también son diferentes para diferentes estaciones del año.
\end{abstract}

El modelo genera cada mes un pronóstico para los tres meses siguientes.

PALABRAS CLAVE: predicción; pronóstico; estacional; MEDSCOPE; índices climáticos.

\section{INTRODUCCIÓN}

La predicción meteorológica a corto plazo ha evolucionado mucho durante las últimas décadas, llegando a ser muy fiable en el plazo de unos pocos días, gracias en gran parte a las mejoras en los modelos meteorológicos de predicción del tiempo. Estos modelos resuelven un problema de condiciones iniciales en el que, fundamentalmente, se predice el estado de la atmósfera y de la superficie terrestre y se consideran el resto de elementos del sistema climático constantes en el tiempo (singularmente la temperatura del agua del mar). Sin embargo, para predicciones a alcances medio y superiores se requiere al menos el acoplo en los modelos del componente oceánico.

Aunque se han hecho grandes avances, especialmente en la predicción a escala estacional sobre los trópicos (hoy día fenómenos como ENSO pueden ser previstos con unos meses de antelación con razonable fiabilidad), los modelos carecen de suficiente pericia a la hora de predecir el carácter de la temperatura y la precipitación en las latitudes medias. Además de las dificultades generales de los modelos para realizar predicciones 
estacionales sobre latitudes medias, la región en torno al mar Mediterráneo resulta especialmente compleja: por un lado, se encuentra en una zona de transición, entre grandes zonas áridas al norte de África, y zonas con clima húmedo, sobre Europa. Por otro lado, presenta una compleja orografía y distribución tierra-océano. Una buena parte de la precipitación anual, además, tiene carácter convectivo en gran parte de la región, por lo que presenta una gran variabilidad, y un único episodio lluvioso puede representar casi la totalidad de las precipitaciones recogidas en una estación.

En este contexto surgen iniciativas como MedCOF, dentro del Marco Mundial de Servicios Climáticos liderado por la OMM, que trata de aunar esfuerzos entre las Asociaciones Regionales I y VI de la OMM para la elaboración de una predicción estacional consensuada utilizando la mayor cantidad posible de fuentes de información. El proyecto MEDSCOPE es parte de la iniciativa ERA4CS de la UE y su principal objetivo es la identificación de fuentes de predecibilidad que mejoren la pericia (skill) de los modelos climáticos utilizados en predicción estacional, así como su aplicación en ciertos sectores sensibles a las condiciones climáticas en escala estacional. Bajo el paraguas de este proyecto, surge la propuesta de elaboración de un modelo empírico de predicción estacional para el Mediterráneo.

\subsection{Elección del modelo empírico}

Dentro del proyecto MEDSCOPE, de manera paralela al desarrollo del Modelo Empírico, está prevista la realización de experimentos de sensibilidad para localizar nuevas fuentes de predecibilidad. El código del modelo empírico se está desarrollando de forma que tenga una gran flexibilidad para introducir cambios tales como la incorporación o eliminación sencilla de predictores. (EDEN et al., 2015) desarrollaron un sistema empírico a nivel global basado en regresión lineal múltiple y que utiliza como predictores unos pocos índices climáticos globales, obteniendo buenos resultados sobre algunas regiones. Utiliza, además, los residuos de la regresión para elaborar una predicción probabilista. (WANG et al., 2017) demuestran, utilizando también un modelo de regresión lineal múltiple, que una elección cuidadosa de los predictores puede proporcionar muy buenos resultados, logrando predecir la NAO de invierno de manera fiable. Este tipo de modelos no requieren de gran potencia de cálculo, y son fácilmente configurables, de manera que el objetivo de poder añadir o eliminar predictores con facilidad resulta factible. La primera versión del modelo empírico aquí descrita y verificada, desarrollada en el marco de MEDSCOPE, sigue la estela de estos dos trabajos. Una segunda versión del modelo incorporará entre otros ingredientes los predictores propuestos y desarrollados por el propio proyecto MEDSCOPE.

\section{MÉTODOS}

\subsection{Definición de regiones geográficas}

Dada la extensión del área estudiada, su gran complejidad (tanto orográfica como por distribución tierra-océano), y su situación geográfica, cada región se ve afectada por diferentes factores en diferentes momentos del año, por lo que, para tratar de mejorar la pericia del sistema, el modelo utiliza diferentes grupos de predictores para cada estación y cada región. Uno de los problemas de este tipo de modelos empíricos es que a veces sus predicciones resultan excesivamente ruidosas, con grandes variaciones entre un píxel y el contiguo. Por tanto, se trata de encontrar un compromiso entre seleccionar los mejores predictores para cada punto, y tratar de que la predicción resulte razonablemente «suave» a escala sinóptica. Para ello se divide el área en estudio en 10 regiones, y se buscan los mejores predictores para cada una de ellas. Para evitar transiciones bruscas en el espacio y en el tiempo, se impone que los predictores de dos regiones vecinas o dos estaciones consecutivas coincidan al menos parcialmente. Además, las regiones se han elegido de manera que exista cierto solape entre ellas cuando son vecinas. La predicción en cada punto de las zonas de solape es el resultado de promediar las predicciones para dicho punto considerado como perteneciente a cada una de las regiones que lo contienen, por separado. 
Las regiones se han definido en base a un análisis de componentes principales sobre el promedio anual de la precipitación, tenido en cuenta solamente las tres primeras funciones empíricas ortogonales (EOF). Cada región se ha seleccionado de modo que contenga alguno de los patrones más marcados y tratando de agrupar por países o zonas geográficas. En la figura 1 se muestran las diferentes zonas escogidas y su representación sobre las tres primeras EOF de precipitación.

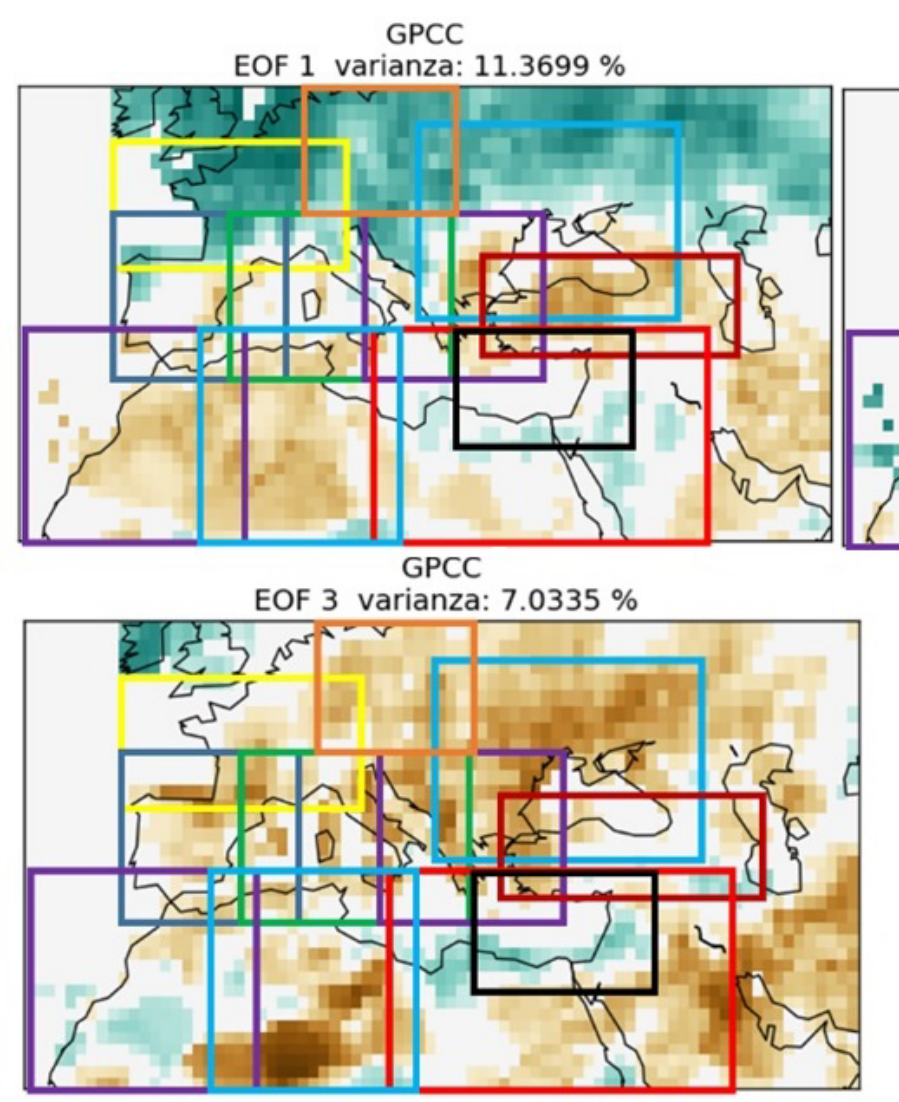

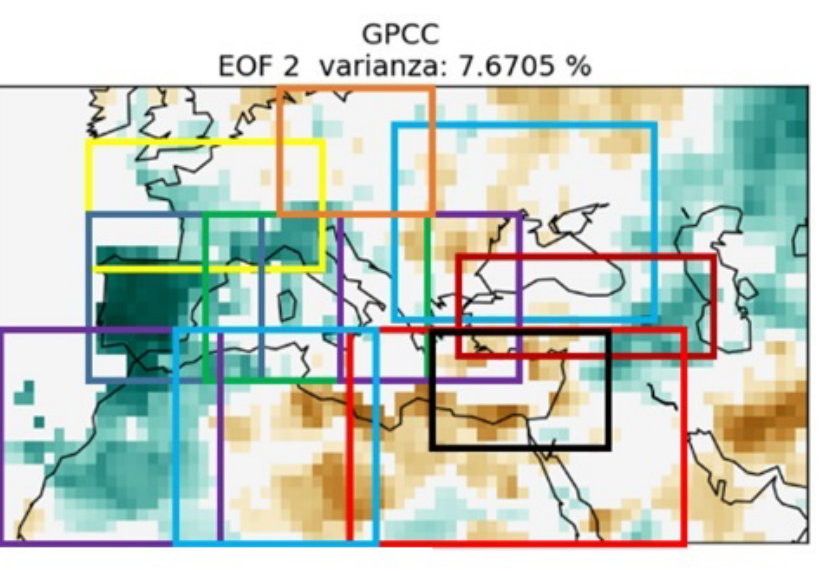

Figura 1. Selección de zonas para ejecutar el modelo empírico frente a las tres primeras EOF de la precipitación (GPCC).

\subsection{Elección de predictandos}

Como predictandos se utilizan inicialmente, para la precipitación, datos del GPCC (SHCNEIDER et al., 2017) en su rejilla de 2,5 grados, y para la temperatura, datos del reanálisis ERA-Interim (DEE et al., 2011) interpolados a 2,5 grados. Los datos de GPCC consisten en una combinación de los datasets GPCC v7 (que comprende desde 1901 hasta 2013) y del dataset de «monitoring» del GPCC (para los años subsiguientes hasta 2017) (https://climexp.knmi.nl/GPCCData/gpcc_25_combined.nc).

Estos datos se proporcionan como valores mensuales. El objeto del modelo es hacer predicciones de cada una de las 12 estaciones del año compuestas por tres meses consecutivos, para lo cual ha de hacer regresiones con el predictando sometido a una media móvil de tres meses. Las series de tiempo resultantes de la media móvil son estandarizadas.

\subsection{Exploración de predictores}

Para configurar el modelo, se utilizan como predictores diferentes índices climáticos globales, establecidos y proporcionados por fuentes externas. Se incluyen patrones globales de teleconexión, índices basados en la temperatura superficial del mar (SST), tanto del Pacífico, como del Atlántico y del Índico, índices de contenido de calor en las primeras capas del océano, e índices de cobertura total de hielo y nieve, formando un total de 25 predictores. Dada la poca señal existente en la zona de interés, se trata de que sea una colección exhaustiva que incremente la probabilidad de que aparezcan al menos unos pocos predictores con una cierta correlación 
para cada zona. Estos índices se han obtenido como dato mensual. Podemos ver estos 25 predictores iniciales en la tabla 1:

\begin{tabular}{|c|c|}
\hline AAO: Antartic Oscillation & Niño3: El Niño Index, region 3 \\
\hline AO: Artic Oscillation & Niño4: El Niño Index, region 4 \\
\hline NAO: North Atlantic Oscillation & $\begin{array}{l}\text { q300: Contenido de calor en los primeros } 300 \text { metros del Pacífico } \\
\text { Ecuatorial }\end{array}$ \\
\hline EA: East Atlantic Pattern & Sn_EuA: Cobertura de nieve en Eurasia \\
\hline EA/WR: East Atlantic/Western Russia & Sn_NA: Cobertura de nieve en Norte América: \\
\hline SCAND: Scandinavia Pattern & Sn_NH: Cobertura de nieve en el hemisferio norte \\
\hline SAM: Southern Annular Mode & DMI: Dipole Mode Index \\
\hline WP: West Pacific Pattern & SEIO: South Eastern Indian Ocean \\
\hline PDO: Pacific Decadal Oscillation & $\begin{array}{l}\text { TNA: Tropical North Atlantic: } \\
\text { https://www.esrl.noaa.gov/psd/data/correlation/tna.data }\end{array}$ \\
\hline MEI: Multivariate ENSO Index & $\begin{array}{l}\text { TSA: Tropical South Atlantic: } \\
\text { https://www.esrl.noaa.gov/psd/data/correlation/tsa.data }\end{array}$ \\
\hline SOI: Southern Oscillation Index & $\begin{array}{l}\text { TASI: Calculado de ERSSTv5, NTA (promedio 20W-40W, } \\
\text { 5N-20N) - SAT (promedio 15W-5E, 20S-5S) }\end{array}$ \\
\hline Niño12: El Niño Index, region 1+2 & $\begin{array}{l}\text { WHWP: Western Hemisphere warm Pool } \\
\text { https://www.esrl.noaa.gov/psd/data/correlation/whwp.data }\end{array}$ \\
\hline Niño34: El Niño Index, region 3,4 & \\
\hline
\end{tabular}

Tabla 1. Predictores iniciales. Los predictores cuyo origen no se ha especificado han sido descargados de https://climexp.knmi.nl/

A partir de los 25 predictores iniciales se obtienen otros nuevos que consisten en el incremento de aquellos, es decir, el valor del nuevo predictor en cada mes es la diferencia entre el valor del predictor original en ese mes y el valor que tenía en el mes anterior. De estos 50 predictores se calcula la anomalía estandarizada de cada dato mensual, respecto de los valores del índice en todos los años pero en el mismo mes del calendario al que pertenece el dato que estandarizamos. Por último, a partir de estos 50 predictores estandarizados generamos otros que consisten en las medias móviles de hasta seis meses.

A partir de todos los predictores así obtenidos (estandarizados y sometidos a media móvil), se hace una selección en base a la correlación lineal que cada uno de ellos tiene con el predictando (media de 3 meses de la estación y estandarizado), aplicando desfases entre ambos de entre 2 y 12 meses. Esto se hace así porque, la ejecución del modelo para los meses $x, x+1, x+2$ se hará en el mes $x-1$, en el que solamente tendremos valores mensuales de los predictores hasta $x-2$. El periodo utilizado para calcular las correlaciones comprende los años 1979-2017.

En cada región, se seleccionan los predictores que muestran una señal más consistente. El modo de hacerlo se detalla a continuación.

En cada región, se obtiene el porcentaje de puntos válidos (hay regiones sin dato por encontrarse en el mar) con una correlación mayor que la significativa (con un nivel de significación del 90\%). Los resultados se representan en una tabla con un formato como el que se muestra en la figura 2. Posteriormente, consultando las tablas de las diferentes zonas, se realiza una selección de predictores tratando de mantener una cierta continuidad, como se mencionó anteriormente, y de que no exista colinealidad entre los predictores de la misma zona y estación. El resultado es una tabla con los predictores que se introducirán en el modelo de regresión lineal múltiple (figura 3). 


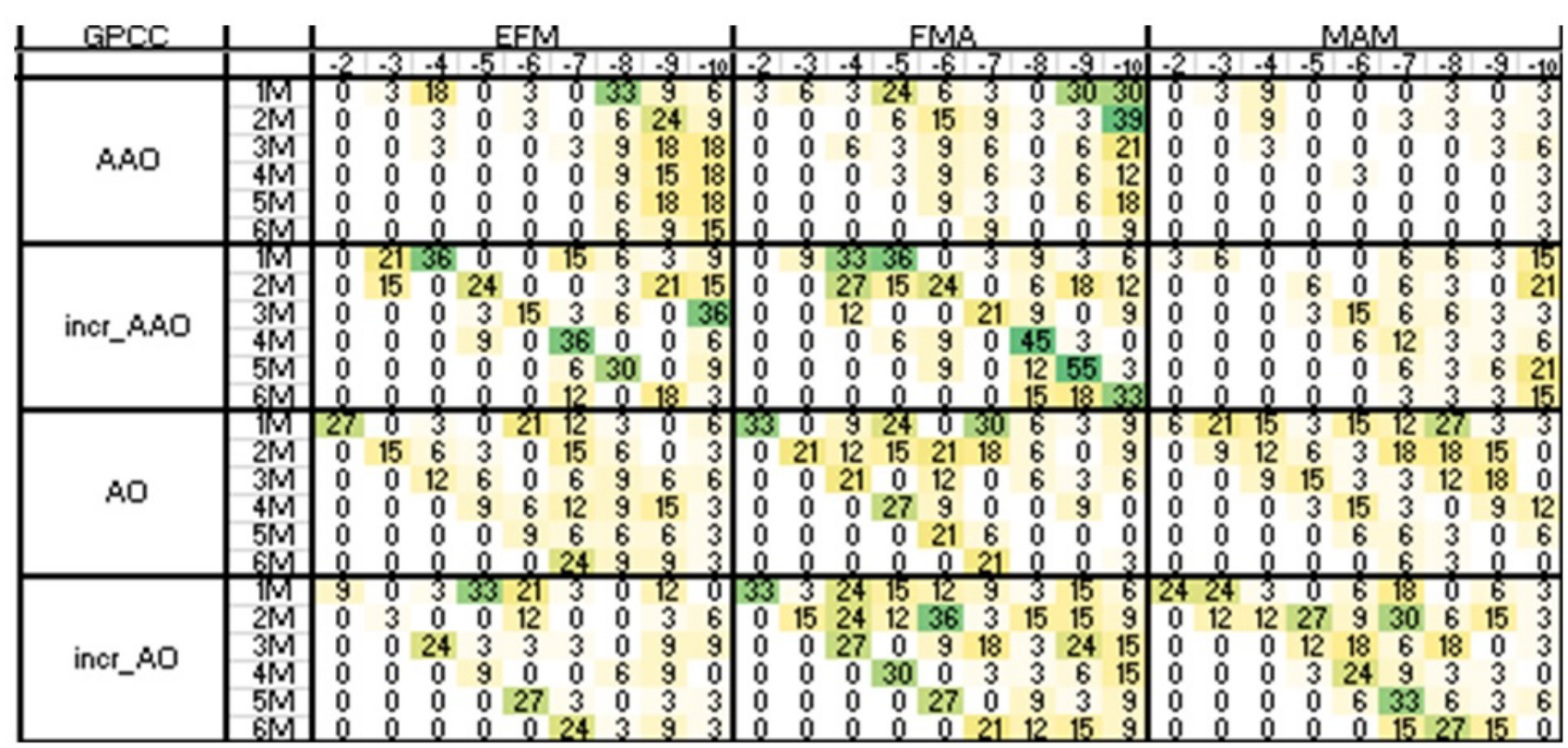

Figura 2. Extracto de la tabla de correlación para una de las zonas. A la izquierda aparece el predictor en cuestión y la media móvil, y arriba el trimestre para el que se correlaciona con la precipitación y el desfase del predictor.

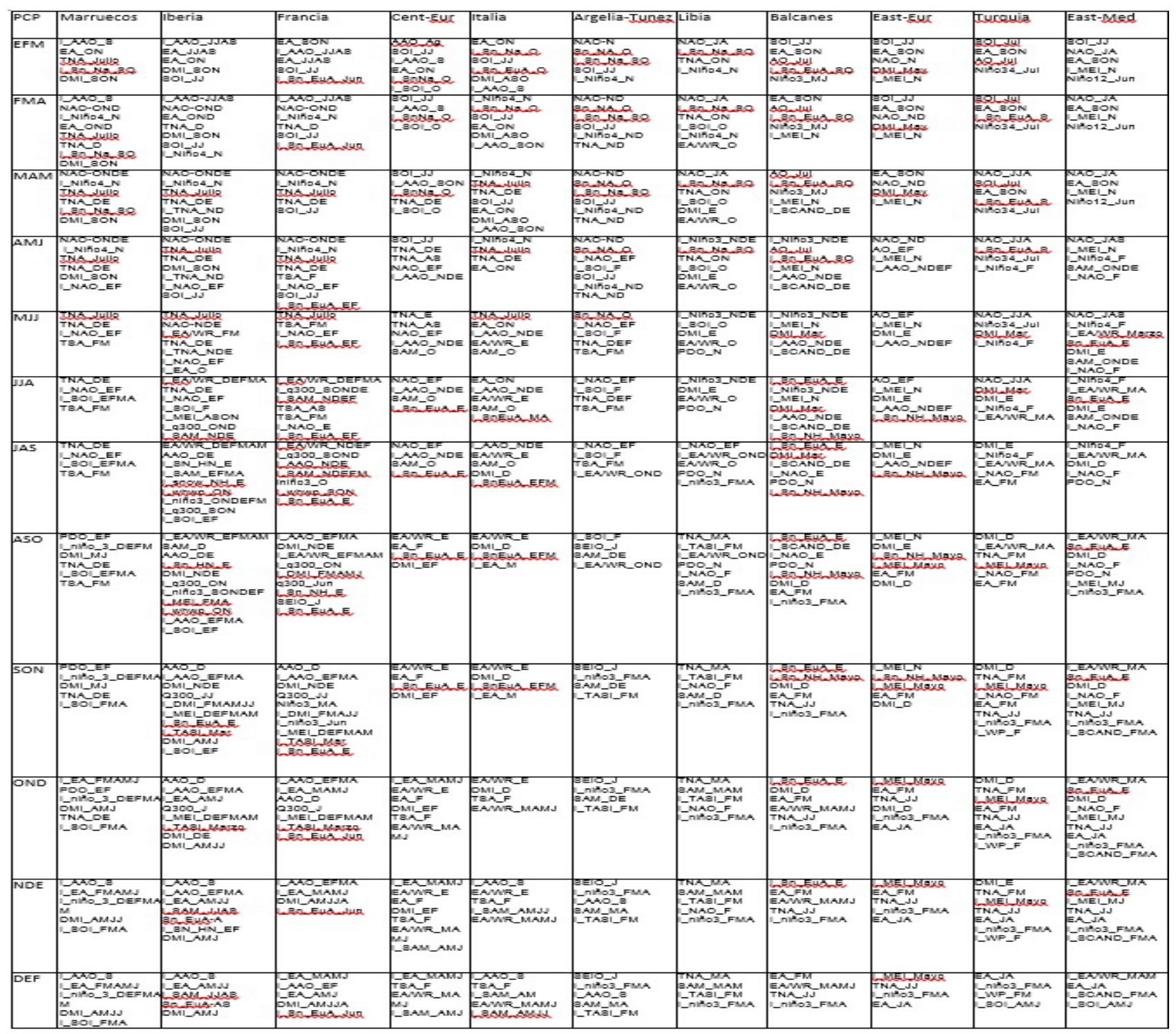

Figura 3. Predictores introducidos en la versión preliminar del modelo, para cada zona y cada trimestre. 


\subsection{Elaboración de predicciones: modelo de regresión lineal múltiple}

El modelo realiza una regresión lineal múltiple (WILKS, 2006 y PASQUI, 2007) en cada punto de grid utilizando los predictores de la región a la que este pertenece y que se han obtenido según se ha descrito en el apartado 2.3. Se deben cumplir ciertos requerimientos para que la regresión lineal múltiple funcione bien (no multicolinealidad de predictores, distribución normal de los residuos, etc.). El modelo hace una validación directa de algunos de estos requisitos: test de Filliben (WILKS, 2006) de la normalidad de los residuos, test de Breusch and Godfrey de la no autocorrelación de los residuos (BREUSCH, 1978 y GoDFrEy, 1978) y detección de multicolinealidad mediante el número de condición de la matriz de predictores. Es de destacar que los residuos de la regresión deben estar normalmente distribuidos. Este requerimiento se consigue satisfacer cuando el predictando tiene distribución normal. En el caso de la lluvia, que no tiene esta distribución, se aplica la raíz cuadrada (PASQUI et al., 2007) para obtener un predictando con una distribución «suficientemente» normal. Antes de la regresión, la tendencia debe ser eliminada tanto de predictores como de predictandos. Más tarde, a la predicción que se obtiene se vuelve a añadir la misma tendencia.

El modelo proporciona tanto una predicción determinista como una predicción por terciles. Estos se calculan a partir de la climatología del predictando transformado. Como, por otra parte, la regresión lineal nos proporciona la distribución (normal) de la predicción (transformada), basta con comparar esta con los terciles previamente calculados para obtener la predicción de probabilidad de cada tercil.

\subsection{Verificación de los resultados}

Con objeto de realizar las primeras comprobaciones del funcionamiento del modelo y para poder realizar una verificación que permita comparar los resultados con los de los modelos climáticos globales utilizados en predicción estacional, se realiza una retropredicción para el período 1983-2014. La regresión se entrena para este período de años, utilizando el método «Leave-One-Out» (WILKs, 2006) con un radio de exclusión de dos años para evitar autocorrelaciones. En la sec. 3.3 se muestran resultados de varios índices de verificación calculados para esta versión del sistema empírico, comparado con los correspondientes para seis sistemas de predicción basados en modelos dinámicos.

\section{RESULTADOS}

\subsection{Predicción de precipitación}

En la figura 4 se muestran algunos ejemplos de predicciones realizadas por el modelo. Aunque se observa algo de ruido en algunas zonas, en general los patrones que se aprecian son de escala sinóptica y razonablemente suaves, por lo que el objetivo de «suavidad» de las predicciones parece cumplirse.

En este caso, además, hemos representado la predicción para el próximo verano (julio-agosto-septiembre), que coincide aproximadamente con la tendencia expuesta por la mayoría de los modelos, o con predicciones como la de MedCOF (aunque esta última es para JJA).

\subsection{Predicción de temperatura}

Utilizando los mismos predictores encontrados para la precipitación, se ejecuta el modelo utilizando la temperatura como predictando, para comparar resultados. 

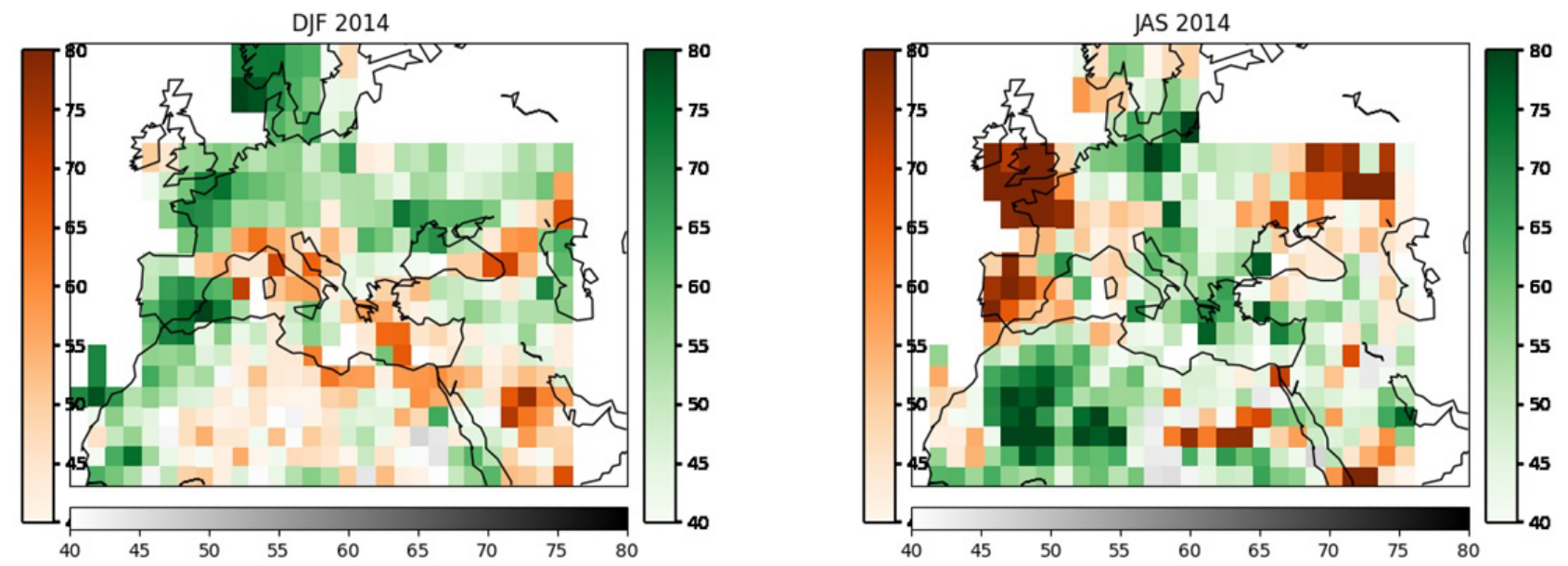

Figura 4. Ejemplos de predicción de precipitación realizadas por el modelo.

En verde la probabilidad del tercil superior, en rojo del inferior, y en gris del tercil central.
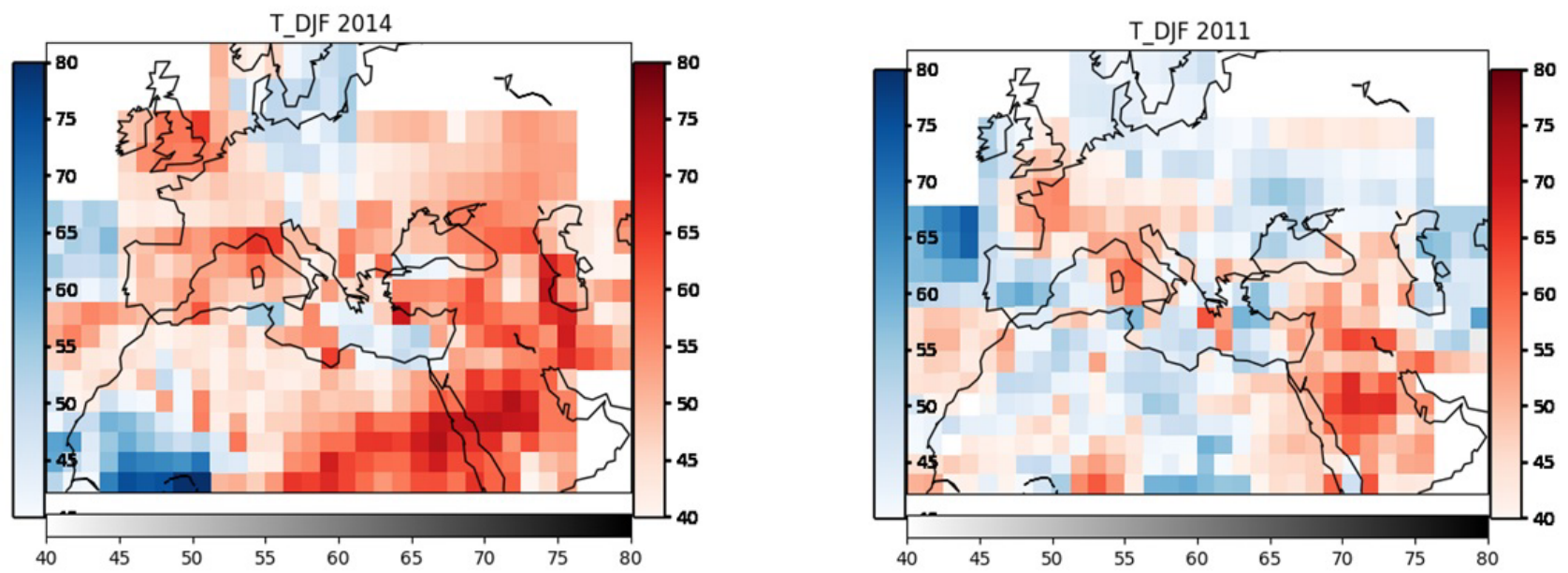

Figura 5. Ejemplos de predicción de temperatura realizadas por el modelo.

En rojo la probabilidad del tercil superior, en azul del inferior, y en gris del tercil central.

\subsection{Verificación}

Para comprobar la pericia de esta primera versión del modelo, se realiza una retropredicción para el período 1997-2009, y se evalúa esta pericia de las predicciones con diferentes índices de verificación: Ranked Probability Skill Score (RPSS) para terciles, y Relative Operating Characteristic (ROC) area y Brier Skill Score (BSS) para dos sucesos (tercil superior o inferior). La definición completa de estos índices puede encontrarse en WILKS (2006). En la figura 6 se representan dos de ellos para precipitación y temperatura, a modo de ejemplo. Los resultados del modelo empírico se comparan con los principales sistemas de predicción estacional basados en modelos dinámicos: ECMWF system 4, Météo-France system 5, UK Met Office system 9 (GloSea5), National Center for Environmental Prediction (NCEP) system version 2, Canadian Seasonal to Inter-annual Prediction System (CanSIPS) and Japanese Seasonal Forecasting System 2 (SÁNCHEZ GARCíA et al., 2018). 
Area: FRANCE Lead-Time: 1 Detrend FALSE / Weighted TRUE

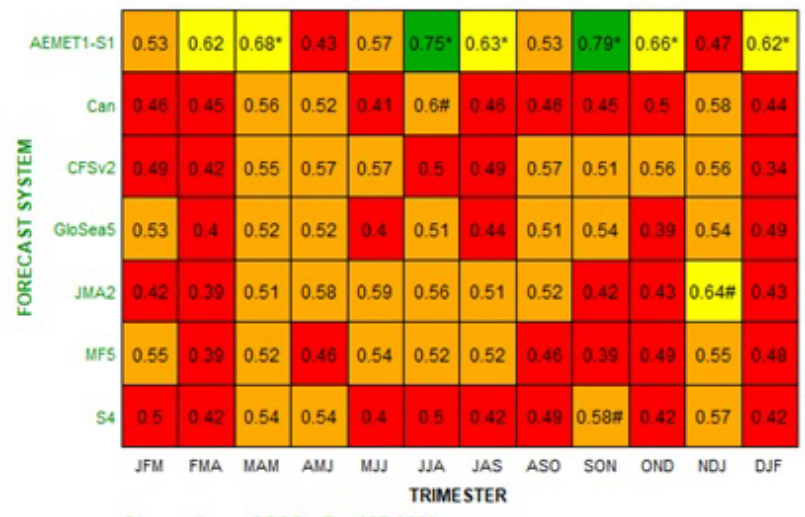

Observations: GPCC_v7 1997-2009

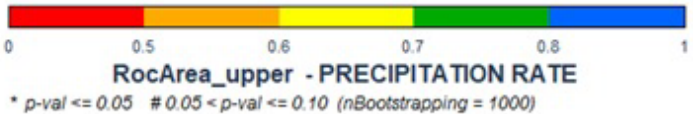

Area: FRANCE Lead-Time: 1 Detrend FALSE/Weighted TRUE

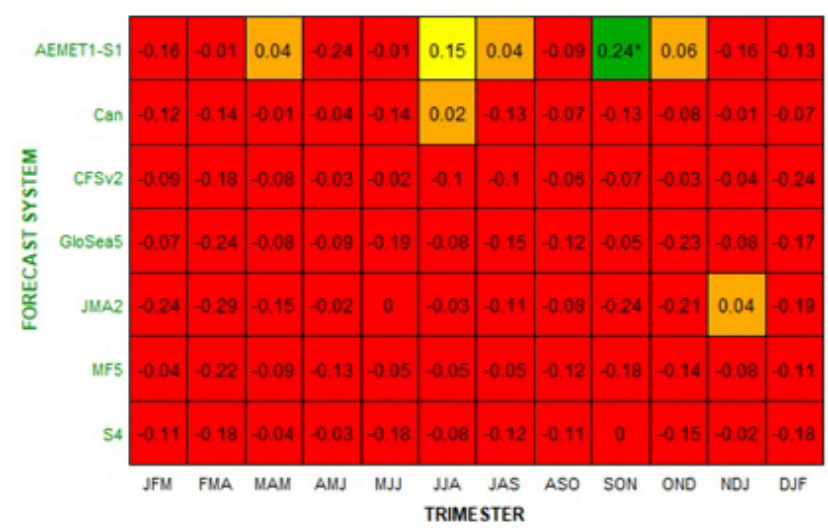

Observations: GPCC_v7 1997-2009

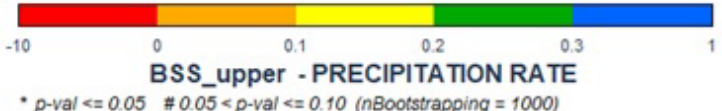

Area: FRANCE Lead-Time: 1 Detrend FALSE / Weighted TRUE

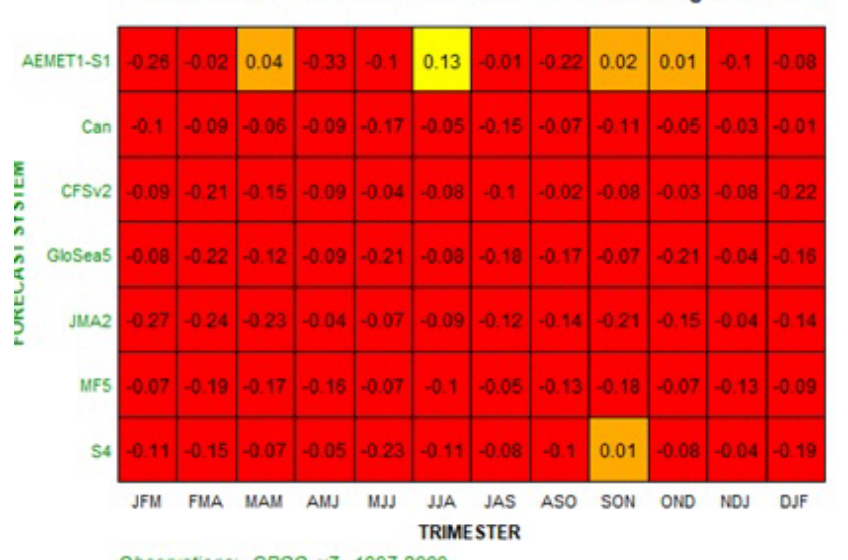

Observations: GPCC_v7 1997-2009

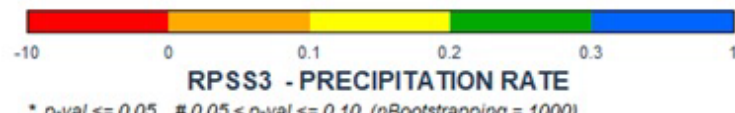

- n-val $<=00.5$ \# $00.5<0$-val $<=010$ inRantotranoing $=1000$
Area: FRANCE Lead-Time: 1 Detrend FALSE / Weighted TRUE

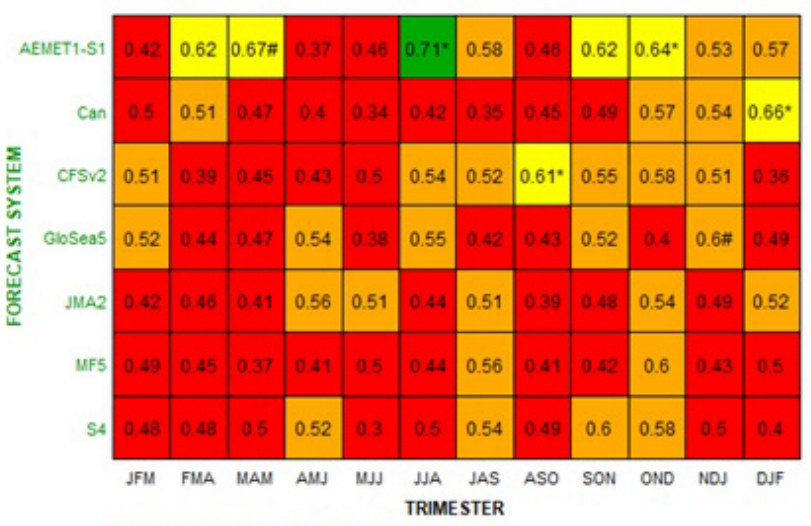

Observations: GPCC_v7 1997-2009

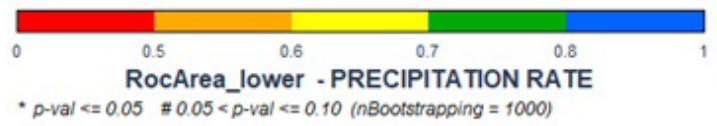

Area: FRANCE Lead-Time: 1 Detrend FALSE/Weighted TRUE

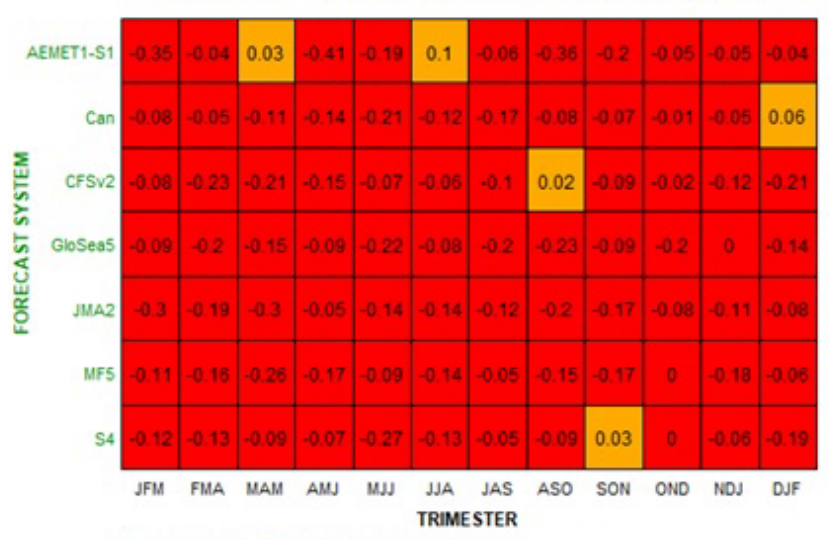

Observations: GPCC_v7 1997-2009

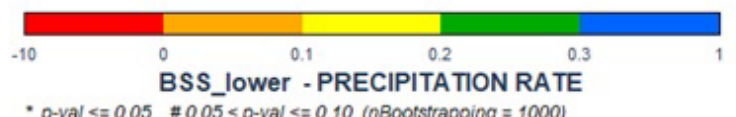

Area: FRANCE Lead-Time: 1 Detrend FALSE / Weighted TRUE

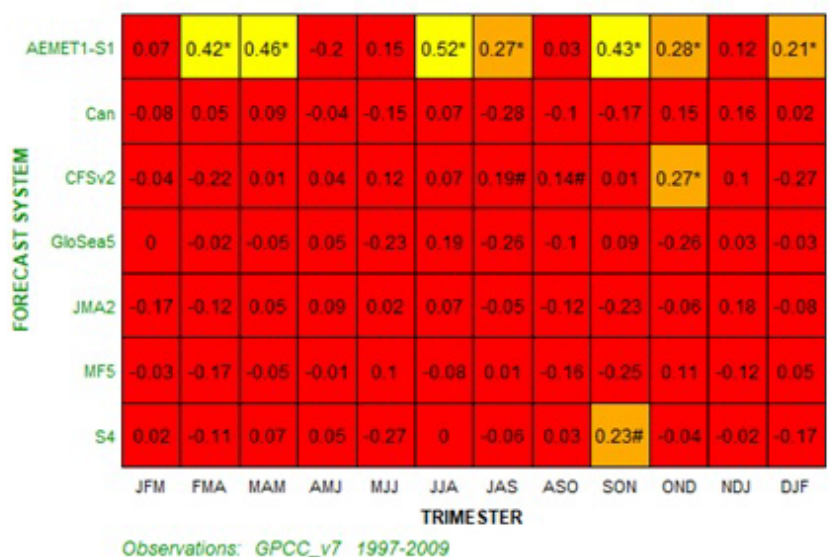

Observations: GPCC_v7 1997-2009

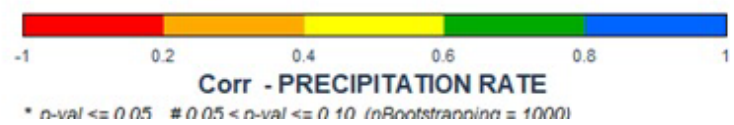

Figura 6.a. Índices de verificación para la precipitación en Francia

(de $10^{\circ} \mathrm{W}$ a $12,2^{\circ} \mathrm{E}$ y de $32,5^{\circ} \mathrm{N}$ a $47,5^{\circ} \mathrm{N}$ ).

Cada columna corresponde a un trimestre sucesivo. 
Area: EAST MED Lead-Time: 1 Detrend FALSE / Weighted TRUE

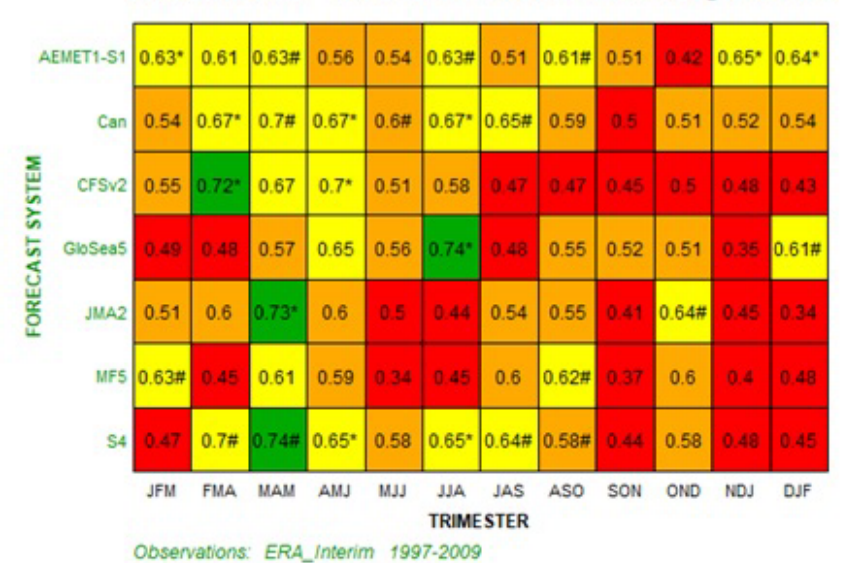

Observations: ERA_Interim 1997-2009

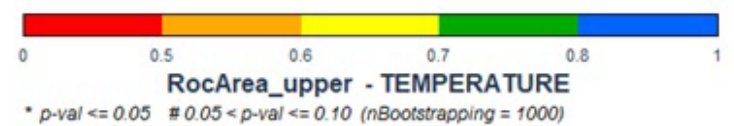

Area: EAST MED Lead-Time: 1 Detrend FALSE / Weighted TRUE

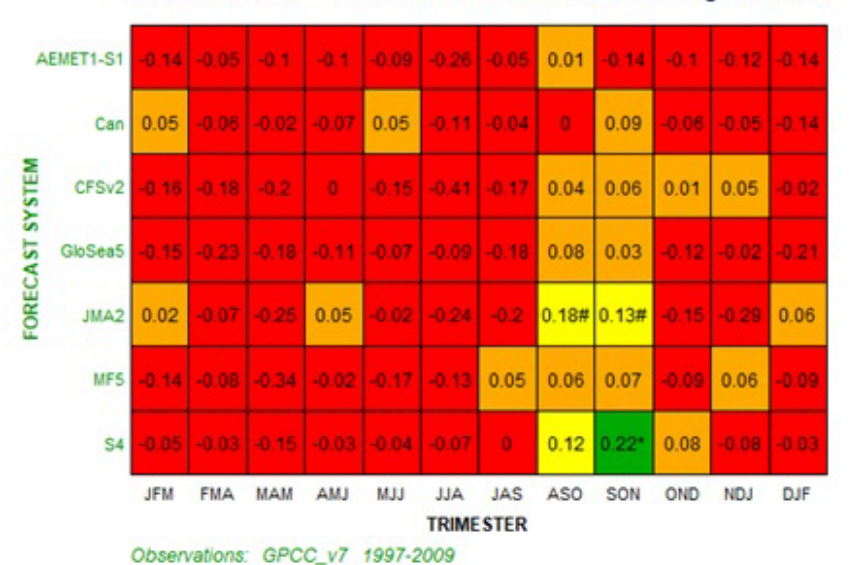

Observations: GPCC_v7 1997-2009

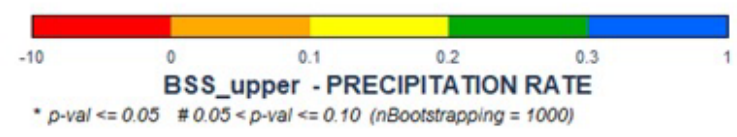

Area: EAST MED Lead-Time: 1 Detrend FALSE / Weighted TRUE

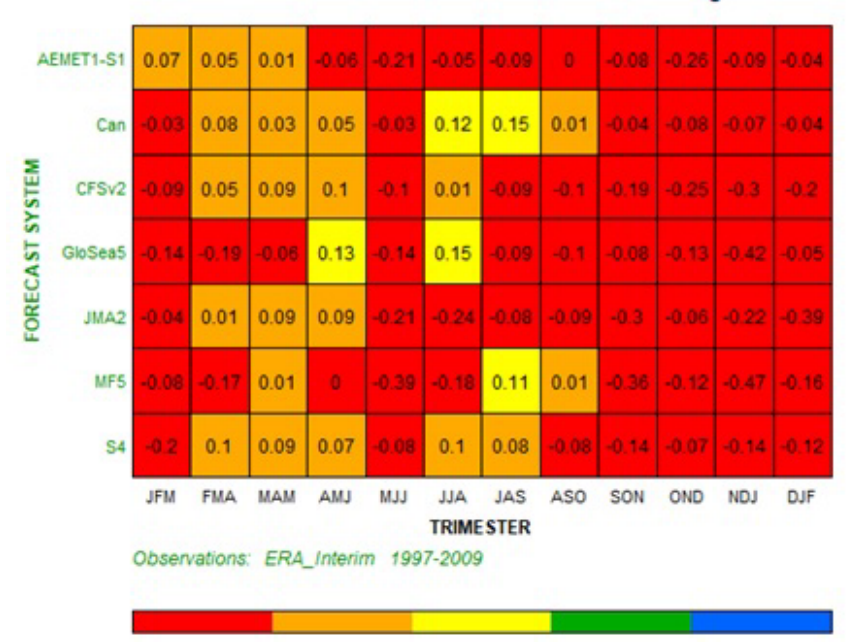

Area: EAST MED Lead-Time: 1 Detrend FALSE / Weighted TRUE

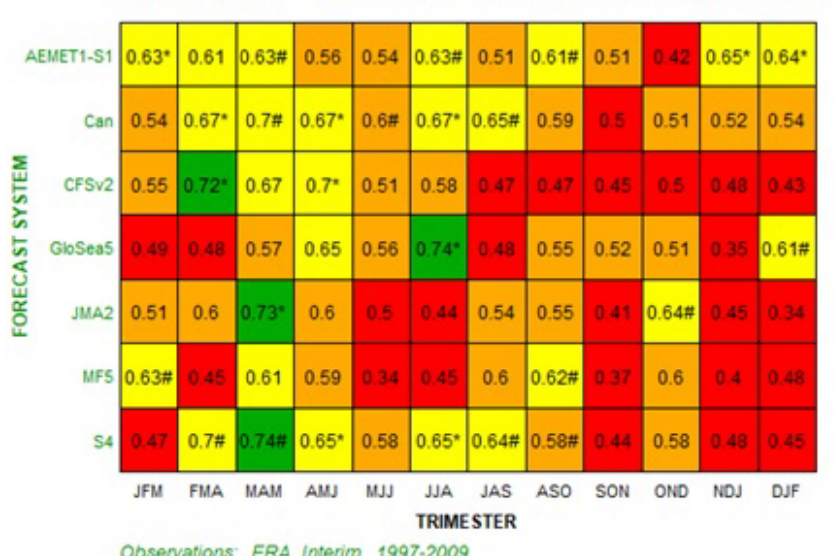

Observations: ERA_Interim 1997-2009

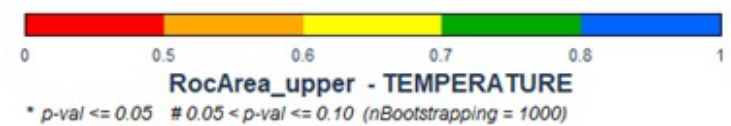

Area: EAST MED Lead-Time: 1 Detrend FALSE / Weighted TRUE

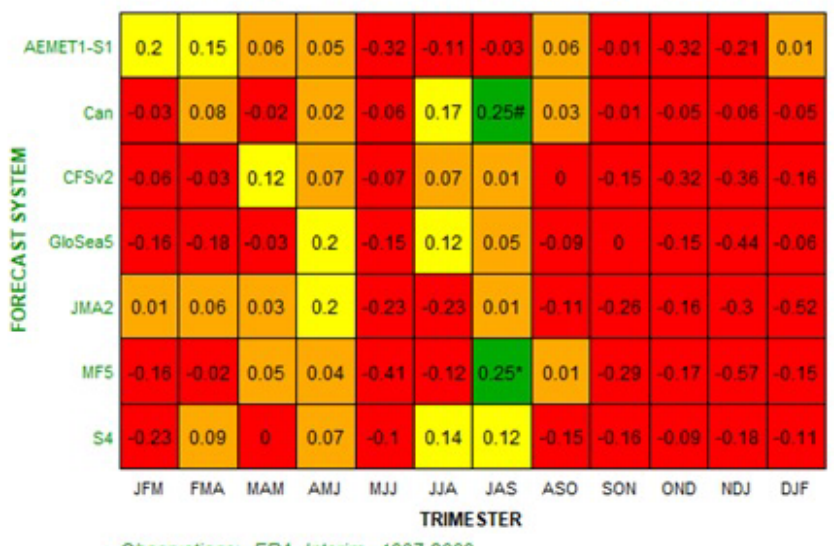

Observations: ERA_Interim 1997-2009

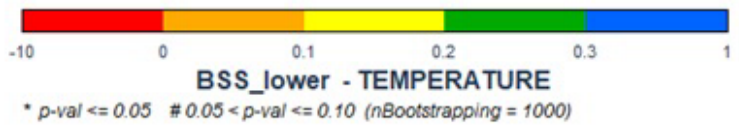

Area: EAST MED Lead-Time: 1 Detrend FALSE / Weighted TRUE

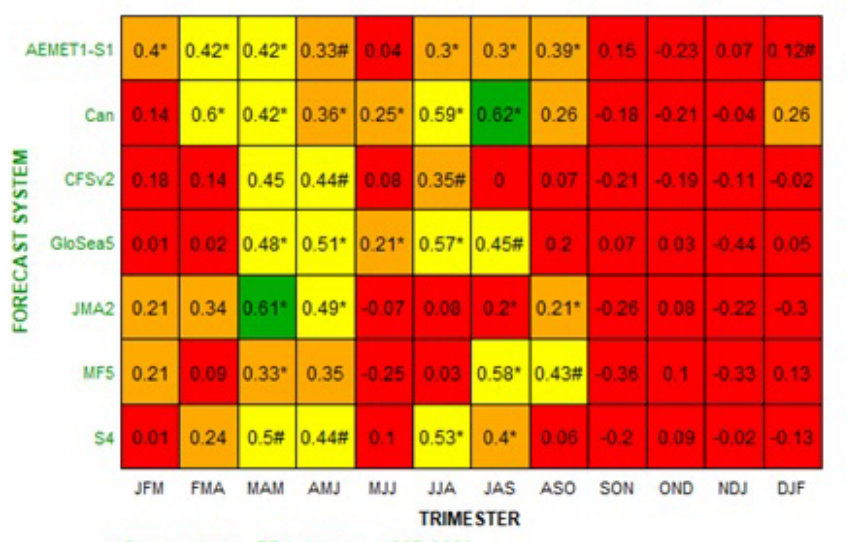

Observations: ERA_Interim 1997-2009

Figura 6.b. Índices de verificación para la temperatura sobre el este del Mediterráneo (de $20^{\circ} \mathrm{E}$ a $40^{\circ} \mathrm{E}$ y de $27,5^{\circ} \mathrm{N}$ a $37,5^{\circ} \mathrm{N}$ ).

Cada columna corresponde a un trimestre sucesivo. 
En las figuras 7 y 8 podemos ver sendos ejemplos para precipitación y temperatura, respectivamente, de la distribución espacial del coeficiente de correlación entre las predicciones del modelo y las observaciones.
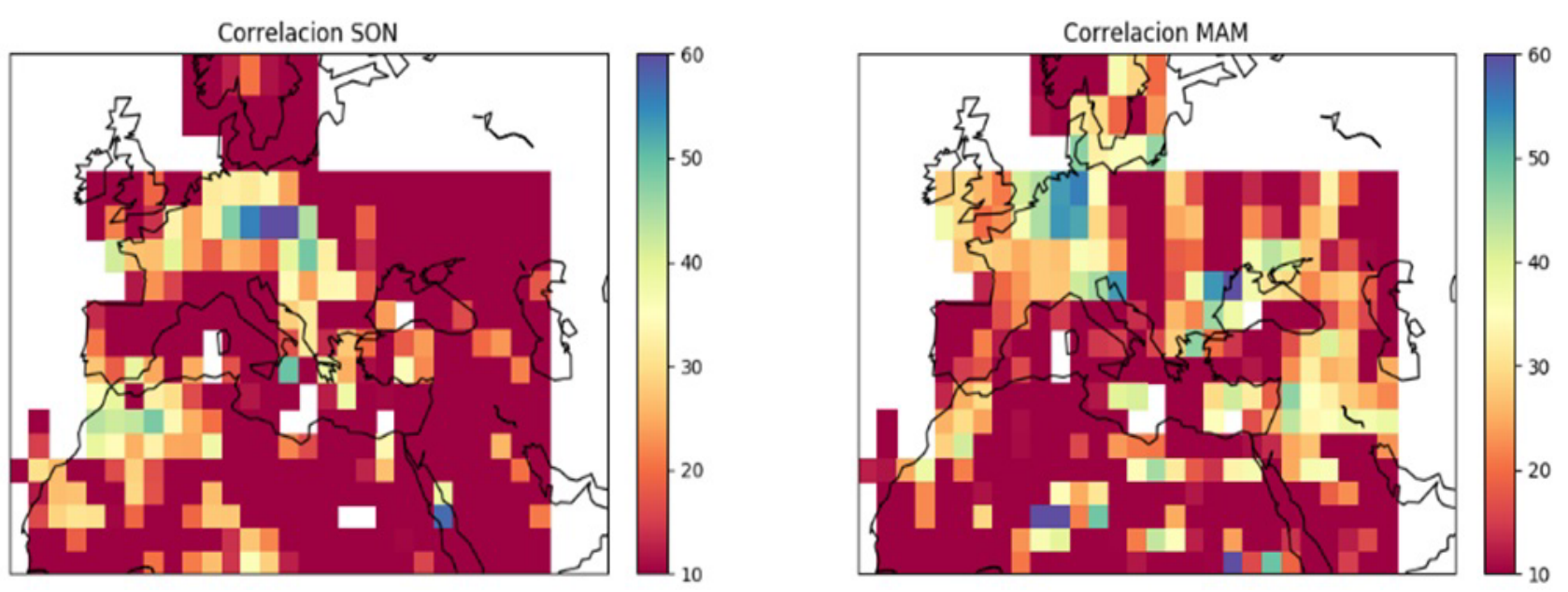

Figura 7. Correlación lineal entre las predicciones del modelo y la observación de precipitación para los meses de septiembre-octubre-noviembre y marzo-abril-mayo.
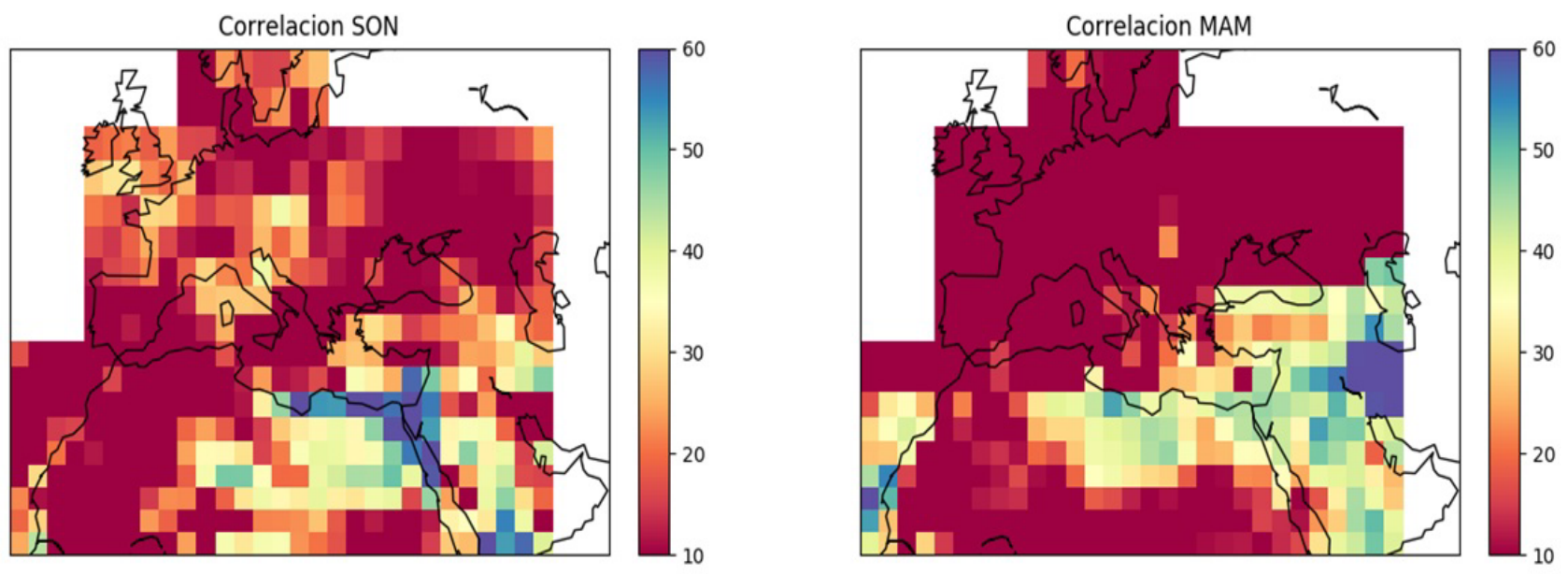

Figura 8. Correlación lineal entre las predicciones del modelo y la observación de temperatura para los meses de septiembre-octubre-noviembre y marzo-abril-mayo.

\section{CONCLUSIONES}

El aspecto general del patrón espacial de las predicciones realizadas con el sistema empírico (figuras 4 y 5) parece cumplir el objetivo de representar estructuras sinópticas y con transiciones suaves en las que no se aprecian las fronteras entre zonas.

La verificación comparada entre el sistema empírico y otros seis sistemas basados en modelos dinámicos utilizando dos índices de verificación (ROC área para el tercil superior y correlación lineal) calculados en un dominio que cubre la península ibérica $\left(36^{\circ} \mathrm{N}-44^{\circ} \mathrm{N}\right.$ y $\left.10^{\circ} \mathrm{W}-5^{\circ} \mathrm{E}\right)$ muestran resultados que en primera instancia son comparables en términos de pericia (fig. 6). Al representar la correlación para dos períodos de tres meses distintos (fig. 7 y 8), pueden verse resultados muy buenos para algunas zonas, mientras que en otras la correlación es muy baja. Queda pendiente de investigar si las diferencias tan apreciables que aparecen en los mapas de correlación de las predicciones dependen del mecanismo elegido para la elección de los predictores o responden a causas más profundas relacionadas con las variaciones geográficas de la predecibilidad. En 
favor de la primera opción está el hecho de que la correlación lineal se calculó entre las series de predictores y la precipitación acumulada. Dado que en el modelo se utiliza la raíz cuadrada de la precipitación para el cálculo de la regresión, y por los mismos motivos que justifican el hacerlo así, habría sido conveniente que el cálculo de correlación se realizara sobre la variable transformada. Además, la selección de predictores para cada zona busca el porcentaje de puntos con correlación significativa, pero no examina su distribución espacial. De hecho, podría haber puntos representados por varios predictores, y otros puntos sin correlación significativa con ninguno de los predictores designados para la zona. Esta selección de predictores se hará de forma automática en la versión definitiva, y la comprobación de este extremo será una de las características que se añadirán al algoritmo.

Por otro lado, para la temperatura se han escogido en esta versión inicial los mismos predictores que para la precipitación. Probablemente puedan obtenerse mejores resultados realizando una exploración de predictores específica para la temperatura, pero el utilizar los mismos predictores para ambas variables tiene la ventaja de poder tratar de asociar las predicciones de ambas variables a las mismas anomalías en la circulación atmosférica y una mejor interpretación física de los resultados.

\section{REFERENCIAS}

Breusch, T. S., 1978. Testing for Autocorrelation in Dynamic Linear Models. Australian. Economic Papers 17 (31), 334-355. http://dx.doi.org/10.1111/j.1467-8454.1978.tb00635.x.

Dee, D. P., Uppala, S. M., Simmons, A. J., Berrisford, P., Poli, P. Kobayashi, S., Andrae, U., Balmaseda, M. A., Balsamo, G., Bauer, P., Bechtold, P., Beljaars, A. C., Van De Berg, L., Bidlot, J., Bormann, N., Delsol, C., Dragani, R., Fuentes, M., Geer, A. J., Haimberger, L., Healy, S. B., Hersbach, H., Hólm, E. V., Isaksen, L., Kållberg, P., Köhler, M., Matricardi, M., Mcnally, A. P., MongeSanz, B. M., Morcrette, J., Park, B., Peubey, C., De Rosnay, P., Tavolato, C., ThéPaut, J. y Vitart, F., 2011. The ERAInterim reanalysis: configuration and performance of the data assimilation system. Q. J. R. Meteorol. Soc., 137: 553-597. doi: http://dx.doi.org/10.1002/qj.828.

Eden, J. M., van Oldenborgh, G. J., Hawkins, E. y Suckling, E. B., 2015. A global empirical system for probabilistic seasonal climate prediction. Geosci. Model Dev., 8, 3947-3973, https://doi.org/10.5194/gmd8-3947-2015.

Godfrey, G., 1978. Testing against general autoregressive and moving average error models when the regressors include lagged dependent variables. Econometrica, 46 (6), 1293-1301.

Pasqui, M., Genesio L., Crisci., Primicerio, J., Benedetti, R. y Maracchi, G., 2007. An adaptive multiregressive method for summer seasonal forecst in the Mediterranean área. 87th AMS, 13-16 January 2007.

Sánchez García, E., Voces Aboy, J. y Rodríguez Camino, E., 2018. Verification of six operational seasonal forecast systems over Europe and Northern Africa. Nota Técnica, AEMET. Puede consultarse en http:// medcof.aemet.es/index.php/models-skill-over-mediterranean.

Schneider, U., Ziese, M., Meyer-Christoffer, A., Finger, P., Rustemeier, E. y Becker, A., 2017. The new portfolio of global precipitation data products of the Global Precipitation Climatology Centre suitable to assess and quantify the global water cycle and resources, Proc. IAHS, 374, 29-34, https://doi.org/10.5194/ piahs-374-29-2016, 2016.

WAng, L., Ting, M. y Kushner, P. J., 2017. A robust empirical seasonal prediction of winter NAO and surface climate, Nature. https://doi.org/10.1038/s41598-017-00353-y.

WILKs, D., 2006. Statistical Methods in the Atmospheric Sciences. Department of Earth and Atmospheric Sciences. Cornell University. 
